\title{
Insight: A genuine factor forimprovementin person with psychosis.
}

\author{
${ }^{1}$ Boban Joseph, ${ }^{2}$ Dr. D. Muralidhar \\ ${ }^{1}$ Ph.D. Scholar, ${ }^{2}$ Professor, ${ }^{1,2}$ Dept. of Psychiatric Social Work National Institute of Mental Health And Neuro \\ Sciences,Bangalore-560029.
}

\section{Introduction}

Understanding precise insight and unawareness of illness in psychosis has been a puzzlefor the researchers and clinicians for a long time.Various attempts were made to understand insight in its cognitive, neurological, cultural and social roots.Insight in cognitive psychologists view is the external attribution about the illness but researchers with psychodynamic approaches found that insight has emotional and intellectual forms base indeed.

According to Amador et al (Amador, 2004) insight is a multi-dimensional construct these includes:(Association, 2000)1.Awareness of mental disorder, 2.Understanding the social consequences of the disorder, 3.Awareness of the need for treatment, 4.Awareness of specific signs and symptoms of the disorder and, 5.The attribution of symptoms to disorder.The classification of insight is based on three aspects 1.Persons with complete insight (aware and correct attributes) 2.Persons being aware of not well and misattributes (aware and misattributes) 3.Those completely not aware of being ill(Roth, Flashman, Saykin, McAllister, \& Vidaver, 2004).

\section{Methods}

The researcher used online original articles for conceptualizing this narrative through Pubmed, Ebsco, Medline, PsycINFO by using the keywords for search:-"insight", OR"awareness to the illness", AND"psychosis", AND"schizophrenia", out of 483 articles after removing duplications of studies and excluding those studies its main variable were not to study and analyses insight. In the final onlinescreening reviewer includedonly 49 studiesof the past 20 years related to insight while considering the inclusion criteria of the review.

The inclusion criteria followed by this narrativeare 1) studies specifically talking about the etiology of insight 2)studies which considers insight as a positive factor in recovery and treatment adherence 3) studies which used validated tools for assessment of insight4) studies explaining about the socio-cultural factors of insight 5) studies correlating cognition and brain function 6) articles published only in English language are included.

Multitude number of researchers attempted in exploring the nature, etiology, predictive and clinical value of the insight in schizophrenia for the last fewdecades, this narrative is attempting to understand the developments in the aspect of insight of patients in psychosis and schizophrenia by the studies in recent couple of decades. Researchers in the field are able to correlate insight with neurological bases, executive functioning, and brain volume and cultural ingredients but, minimal studies found to be looking intothe aspects of improving insight among the affected individuals as using the direct interventions.

A few direct interventions such as motivational interviewing, cognitive behavior therapy are still use to improve the same. These interventions proved to be effective among studies but not given a satisfactory results that itwill improve longstanding insight into a person's illness and related aspects to a great extent. The past studies supporting enough evidence for the assessment of insight and majority of the researches able to develop better understanding of it.Here thisnarrative looking into aspects of insight among persons affected with schizophrenia and what is lacking in the studies related to insight.

\section{Understanding the Insight}

Impaired or lack of insight into ones mental illness is been considered as one of the most noticed symptoms in schizophrenia (Amador, 2004) although among the 12 symptoms; thislack of insight has the greatest capability to differentiate schizophrenia from depression and rest of the psychoses (Carpenter, Strauss, \& Bartko, 1973).About $50 \%$ to $80 \%$ of people affected with schizophrenia have lack of insight to their illness(Lincoln, Lullmann, \& Rief, 2007). 
Insight in to a psychiatric illness has been considered by researchers as a multidimensional fact than a single concept., it covers the awareness of the presence of mental illness, this factor looking into different etiological reasons such as biological, psychopathological, environmental are helpful in understanding insight in psychosis and treatment compliance and the capacity to redefine bizarre and unusual mental events as not normal(David et al., 1995).It also includes attribution and awareness. These dimensions accepted well for conceptualizing the concept insight. Awareness of symptoms to mental illnessis recognition of the illness with social and medical consequences and attribution is the explanation of the patient about the root of the signs and symptoms that they experience(Amador et al., 1993) and the attitudes to the former and later illness(McEvoy, Applebaum, Apperson, Geller, \& Freter, 1989).

Furthermore, although insight can be seen as modality oriented for instance., person with schizophrenia may aware about the some aspects of his or her illness(Amador, Strauss, Yale, \& Gorman, 1991)(Kendler, Glazer, \& Morgenstern, 1983).The Diagnostic and Statistical Manuel considers poor insight is related to schizophrenia than a coping strategy, which can be lead to non-compliance, increased risk of relapse, involuntary hospital admissions, poor psychosocial functions and ultimately poorer prognosis(Association, 2000)

\section{Measurement tools for assessing Insight}

Plenty of the research studies conducted in the past few decades had tried to understand the concepts and various dimensionsof Insight; Nevertheless many measurement scales developed to study the insight and related treatment attitude of the persons with schizophrenia.The commonly used tools are Scale for the Unawareness of Mental Disorder(SUMD)-To assess the current and retrospective insight of the patient(Amador et al., 1993),Insight and Treatment Attitude Questionnaire (ITAQ)- To assess the inability of the patient to recognize the illness and the attitude towards the treatment.

Schedule for Assessment of Insight Expanded (SAI-E). It assesses the insight in three dimensions such as awareness, relabeling of symptoms, and compliance plus the ability to consider it on another's perspective(Kemp R, 1997; Sanz, Constable, Lopez-Ibor, Kemp, \& David, 1998).Birchwood Insight Scale(BIS)-It assesses three areas of insight mainly understanding ones mental illness, capacity to re-label psychotic symptoms, and understanding the need for treatment(Birchwood et al., 1994).

Beck Cognitive Insight Scale(BCIS) is a 15 item self- report scale developed to assess patients individual reflectionabout their experiences and explanationsand greater confidence(Beck, Baruch, Balter, Steer, \& Warman, 2004). Short Explanatory Model Interview (SEMI)-It includes semi-structured open ended questions to understand their concepts held by the patients about the reason for their consultation, name of the problem, perceived causes and consequences, severity, cause and effect on the individuals physique, work, family life, social relations, and emotions(Joel et al., 2003; Lloyd et al., 1998).PANASS is a 30 item scale designed to assess psychopathology related to illness have an insight dimension from clinician's perspectivethat is onpatient's acceptance of illness and lack of judgment(Kay, Fiszbein, \& Opler, 1987; Tranulis, Corin, \& Kirmayer, 2008)

\section{Etiology of Insight}

Poor insight in schizophrenia rests in two approaches: the traditional view says it is related to defense or coping mechanism but the present views mentions about its neurological factors behind it but contemporary studies pinpoint to neuropsychological limitations, these two together contributes to a better base for poor insight into the illness in schizophrenia (Xavier F Amador. \& David, 2004).

The researches in the field somewhat succeeded to bring it to the light that poor insight is completely associated to longer duration of untreated psychosis(de Haan, Peters, Dingemans, Wouters, \& Linszen, 2002)., although studies conducted on insight into illness stick to the multidimensional aspectsof awareness and its attribution about the illness by an affected member and to the psychotic symptoms(Amador, 1998). Though insight has significant effect on patients differential experiences of their disorder and these insights are predominantly connected with global and particular measures of outcome(Schwartz, Cohen, \& Grubaugh, 1997).

Researchersalso found that the influences of neuroanatomical or neuropsychological causes with evidence and apart from that genetics, environmental factors such as education and socio-economic class are connected to schizophrenia which included insight and use of medicines, severity of illness too contributed (Chen et al., 2005)(Cernovsky, Landmark, Merskey, \& Husni, 2004; Gallagher, Jones, McFalls, \& Pisa, 2006).Brain imaging studies found a minimization in the overall size of the brain(Flashman, McAllister, Andreasen, \& Saykin, 2000)and atrophy in the frontal lobe(Laroi et al., 2000)found as insight impaired in patients with schizophrenia.The social factor theory postulates that patient's grades of insight can be affected by social and interpersonal factors(Granholm et al., 2005; Rector \& Beck, 2002; Rusch \& Corrigan, 2002; Valmaggia, van der Gaag, Tarrier, Pijnenborg, \& Slooff, 2005). 
An individual's ability for self-awareness originates from social processes, through social learning and the ways in which one describes about him that are specific to the culture that the individual comes from(Amador \& David, 1998)In addition, the explanatory models of insight brings out that insight should be assessed on the basis of native cultural standards than considering it as universal like medically based concepts of health and illness(Saravanan, David, Bhugra, Prince, \& Jacob, 2005)

\section{Utility of insight in schizophrenia}

Multiple studies of the past decades found the association between insight and quality of life as predictors of outcome in schizophrenia (Addington \& Addington, 2008; Matsui, Sumiyoshi, Arai, Higuchi, \& Kurachi, 2008; Woon, Chia, Chan, \& Sim, 2010).Researches' demonstrates that insight is helpful in improving adherence to medication at one level and to some extent it reduces the negative effect on stigmatization by segregating the illness from ones identity this will be stable with the idea of recovery. The insight can be used to separate the symptoms of the illness from genuine reality and from self-identity(Lincoln et al., 2007).

Studies indicate that insight to be significantly related to positive symptoms(Amador et al., 1994; Baier et al., 2000; Collins, Remington, Coulter, \& Birkett, 1997; De Hert et al., 2009; Ritsner \& Blumenkrantz, 2007; Stefanopoulou, Lafuente, Saez Fonseca, \& Huxley, 2009) negative symptoms(Amador et al., 1994; Cuesta, Peralta, \& Zarzuela, 1998; Danki, Dilbaz, Okay, \& Telci, 2007; De Hert et al., 2009; Mintz, Addington, \& Addington, 2004; Monteiro, Silva, \& Louza, 2008; Ritsner \& Blumenkrantz, 2007; Simon, Berger, Giacomini, Ferrero, \& Mohr, 2006; Smith, Hull, Israel, \& Willson, 2000; Stefanopoulou et al., 2009) the positive and negative symptoms together(Debowska, Grzywa, \& Kucharska-Pietura, 1998; Mintz, Dobson, \& Romney, 2003)disorganized symptoms(Baier et al., 2000; Dickerson, Boronow, Ringel, \& Parente, 1997; Smith et al., 2000 ) in absence of symptoms (Schwartz \& Petersen, 1999)and during the early period of the illness(Keshavan, Rabinowitz, DeSmedt, Harvey, \& Schooler, 2004).

Studies also found evidence that during acute episodes of illness the relation between positive symptoms and insight is high in relation with periods of remission (Lincoln et al., 2007; Mintz et al., 2003; Sevy, Nathanson, Visweswaraiah, \& Amador, 2004; Tirupati, Padmavati, Thara, \& McCreadie, 2007).Family members of the affected individual notably agree that insight into a mental illness is a beginning of the adherence to treatment, recovery and rehabilitation for persons with the kind of disability(McEvoy, 2004)(Frese, 2000 ) but the same time they share that getting insight to ones schizophrenia worsen it too (L.J \& Korin.E, 1998).The recent study by(Kurtz, Olfson, \& Rose, 2013) found that a precise insight into ones illness is an indispensable factor for determining self-efficacy and poor insight into the illness would affect a person's ability to complete tasks and reach goals.

\section{Socio-cultural believes in understanding Insight}

Social and cultural aspects play a major contributor in the development of insight in a person towards the mental illness and its treatment, these socio-cultural factors can lay foundation within a person to form believes to explain their illness will be probably valid(Kleinman., 1980). The multiple dimensions of insight is variously affected by disease/socio-cultural factors(Gigante \& Castel, 2004). Research in all over the world pointing that the symptoms, help seeking, course of schizophrenia these are influenced along with the other psychiatric disorders and their cultural explanations.

Explanatory models talks about non-medical beliefs such as black magic, evil spirit, karma, sin and punishment by God or supernatural powers, than medical concepts for instance nutritional deficiency, degeneration, disease. This is found more in low and middle income countries however also found in western populations (Lloyd et al., 1998; McCabe \& Priebe, 2004).Clinicians assessments found that socio cultural factors have major effect on the insight in patients with schizophrenia(Tranulis et al., 2008). The changing world due to globalization has an impact on culture of India compare to the west and it has an effect on belief models in schizophrenia which is assimilating the western biomedical models incorporating various models also would be beneficial for the mentally ill in developing and undeveloped worlds(Saravanan et al., 2007).

\section{Insight and Cognition}

Various studies speculate that poor insight is related to cognitive deficits(Monteiro et al., 2008; Ritsner \& Blumenkrantz, 2007; Shad, Muddasani, Prasad, Sweeney, \& Keshavan, 2004)(Drake \& Lewis, 2003; Rossell, Coakes, Shapleske, Woodruff, \& David, 2003; Smith et al., 2000). Results of a study found that patients with better insight into the clinical symptoms had better executive functioning(Medalia \& Thysen, 2010) and patients poor insight has associated with deficits in executive functioning(Drake \& Lewis, 2003; Lysaker, Whitney, \& Davis, 2006; Rossell et al., 2003) mainly with abstract measuring and mental flexibility and selfreflection(David, 1990) but few studies found no relationship with this variables(Goldberg, Green-Paden, Lehman, \& Gold, 2001) however, although some other studies found factors that contributes awareness to mental illness along with executive functioning is verbal memory-learning among patients with schizophrenia. 
Another study found relationship between insight and cognition and emotional function, in that convincing evidence related to visual object learning, identification of facial emotions, and verbal working memory and finally authors concluded that poor insight related to cognitive impairment but not particular to executive function in chronic schizophrenia(Goodman, Knoll, Isakov, \& Silver, 2005).

\section{Insight and Brain Function}

Theories postulates neurological basis for insight schizophrenia, neuropsychological tests related to frontal lobe functioning is found assuring it(Drake \& Lewis, 2003; Lysaker, Bell, Bryson, \& Kaplan, 1998). Clinical symptoms and common neuropsychological impairment found to be linked (Aleman, Agrawal, Morgan, \& David, 2006)with brains frontal lobe functions(Amador et al., 1993; Lysaker \& Bell, 1994; Young, Davila, \& Scher, 1993) these frontal lobe functioning has relation to insight into clinical symptoms(Medalia \& Thysen, 2010).

A study on antipsychotic drug naïve patients in first episode schizophrenia were examined the awareness and attribution of symptoms on neuro-anatomy of prefrontal sub regions and found that right dorsolateral prefrontal cortex was correlated with average scores of awareness of symptoms and right medial orgitofrontal cortex correlated with average scores on present attribution of symptoms (Shad, Muddasani, \& Keshavan, 2006).

\section{Conclusion}

The ability of a person to recognize the changes in him/her mind or body which affects his/her mental and social functioning and the same time recognizes the need for restoration of that ability and compliance of treatment which is called "Insight." Insight is studied at various levels by researchers in biological, psychological and psychosocial, cultural perspectives and has given various terms for labeling it such as anosognosia or unawareness of neurological deficits, lack of awareness, absence, or denial at different perspectives.

Treatment options to improve insight are minimal, and not many interventions developed to look into improving aspects of insight in direct mode but few intervention namely cognitive skills training and psychoeducation(Nieznanski, Czerwinska, Chojnowska, Walczak, \& Dunski, 2002) motivational interviewing and cognitive behavior therapy(Silver, 2003) has been used as an adjunct mode of effective options for improving insight as part of treatment(Granholm et al., 2005; Rector \& Beck, 2002; Rusch \& Corrigan, 2002; Valmaggia et al., 2005) and few studies found insight shall be improved as part of enhancement in cognition and executive functioning. But it is found in a study that these form of treatment will help those who misattribute their symptoms and are aware of their illness(Mysore et al., 2007) However but only a very few studies looked into interventions directly pointing to improve insight. Innovative and integrating different treatment strategies should be developed to cater the need for improving insight.

Moreover, from this narrative author conclude that a precise need for studies looking at insight in person with severe mental illness in their prodromal states of psychosis, early stages/episodes of psychosis, and persons with never treated psychosis or schizophrenia and has major implications for management and preventing relapse.In addition to that the recent longitudinal studies of South India found the relevance to sociocultural beliefs and the different modes of help seeking behavior also found to be relevant to certain kind of the presence of insight. In addition;there is also a gap for intervening multi-disciplinary team such as psychiatric social workers, psychologists and psychiatric nurses to insight researchfor exploringeffective interventions on insight at a large extent bykeeping in mind to understand the socio-cultural beliefs.

\section{Conflict of interest}

The author does not have come across any conflict of interest while doing the narrative review.

\section{References}

[1]. Addington, J., \& Addington, D. (2008). Social and cognitive functioning in psychosis. Schizophr Res, 99(1-3), 176-181. doi: 10.1016/j.schres.2007.07.004

[2]. Aleman, A., Agrawal, N., Morgan, K. D., \& David, A. S. (2006). Insight in psychosis and neuropsychological function: metaanalysis. Br J Psychiatry, 189, 204-212. doi: 10.1192/bjp.189.3.204

[3]. Amador, X. F. (1998). The description and meaning of insight in psychosis. New York: Oxford University Press.

[4]. Amador, X. F., \& David, A. S. (1998). Inside knowledge - cultural construction of insight in psychosis. In C. E. Kirmayer L.J (Ed.), Insight and Psychosis. (pp. 193-220). New York: Oxford University Press.

[5]. Amador, X. F., Flaum, M., Andreasen, N. C., Strauss, D. H., Yale, S. A., Clark, S. C., \& Gorman, J. M. (1994). Awareness of illness in schizophrenia and schizoaffective and mood disorders. Arch Gen Psychiatry, 51(10), 826-836.

[6]. Amador, X. F., Strauss, D. H., Yale, S. A., Flaum, M. M., Endicott, J., \& Gorman, J. M. (1993). Assessment of insight in psychosis. Am J Psychiatry, 150(6), 873-879.

[7]. Amador, X. F., Strauss, D. H., Yale, S. A., \& Gorman, J. M. (1991). Awareness of illness in schizophrenia. Schizophr Bull, 17(1), 113-132. 
[8]. Amador, X. F. D. A. S. (2004). Insight and Psychosis:Awareness of illness in Schizophrenia and related disorders. Oxford: Oxford University Press, (2nd edn).

[9]. Association, A. P. (2000). Diagnostic and Statistical Manual of Mental Disorders, Fourth Edition: DSM-IV-TR®: American Psychiatric Association.

[10]. Association, A. P. (2000). Diagnostic and Statistical Maual-IV (Vol. Text Revison-IV). Washigton DC: American Psychiatric Association.

[11]. Baier, M., DeShay, E., Owens, K., Robinson, M., Lasar, K., Peterson, K., \& Bland, R. S. (2000). The relationship between insight and clinical factors for persons with schizophrenia. Arch Psychiatr Nurs, 14(6), 259-265. doi: 10.1053/apnu.2000.19088

[12]. Beck, A. T., Baruch, E., Balter, J. M., Steer, R. A., \& Warman, D. M. (2004). A new instrument for measuring insight: the Beck Cognitive Insight Scale. Schizophr Res, 68(2-3), 319-329. doi: 10.1016/s0920-9964(03)00189-0

[13]. Birchwood, M., Smith, J., Drury, V., Healy, J., Macmillan, F., \& Slade, M. (1994). A self-report Insight Scale for psychosis: reliability, validity and sensitivity to change. Acta Psychiatr Scand, 89(1), 62-67.

[14]. Carpenter, W. T., Jr., Strauss, J. S., \& Bartko, J. J. (1973). Flexible system for the diagnosis of schizophrenia: report from the WHO International Pilot Study of Schizophrenia. Science, 182(4118), 1275-1278.

[15]. Cernovsky, Z. Z., Landmark, J. A., Merskey, H., \& Husni, M. (2004). Clinical correlates of insight in schizophrenia. Psychol Rep, 95(3 Pt 1), 821-827.

[16]. Chen, K. C., Chu, C. L., Yang, Y. K., Yeh, T. L., Lee, I. H., Chen, P. S., \& Lu, R. B. (2005). The relationship among insight, cognitive function of patients with schizophrenia and their relatives' perception. Psychiatry Clin Neurosci, 59(6), 657-660. doi: $10.1111 / \mathrm{j} .1440-1819.2005 .01433 . \mathrm{x}$

[17]. Collins, A. A., Remington, G. J., Coulter, K., \& Birkett, K. (1997). Insight, neurocognitive function and symptom clusters in chronic schizophrenia. Schizophr Res, 27(1), 37-44. doi: 10.1016/s0920-9964(97)00075-3

[18]. Cuesta, M. J., Peralta, V., \& Zarzuela, A. (1998). Psychopathological dimensions and lack of insight in schizophrenia. Psychol Rep, 83(3 Pt 1), 895-898.

[19]. Danki, D., Dilbaz, N., Okay, I. T., \& Telci, S. (2007). [Insight in schizophrenia: relationship to family history, and positive and negative symptoms]. Turk Psikiyatri Derg, 18(2), 129-136.

[20]. David, A., van Os, J., Jones, P., Harvey, I., Foerster, A., \& Fahy, T. (1995). Insight and psychotic illness. Cross-sectional and longitudinal associations. Br J Psychiatry, 167(5), 621-628.

[21]. David, A. S. (1990). Insight and psychosis. Br J Psychiatry, 156, 798-808.

[22]. de Haan, L., Peters, B., Dingemans, P., Wouters, L., \& Linszen, D. (2002). Attitudes of patients toward the first psychotic episode and the start of treatment. Schizophr Bull, 28(3), 431-442.

[23]. De Hert, M. A., Simon, V., Vidovic, D., Franic, T., Wampers, M., Peuskens, J., \& van Winkel, R. (2009). Evaluation of the association between insight and symptoms in a large sample of patients with schizophrenia. Eur Psychiatry, 24(8), 507-512. doi: 10.1016/j.eurpsy.2009.04.004

[24]. Debowska, G., Grzywa, A., \& Kucharska-Pietura, K. (1998). Insight in paranoid schizophrenia--its relationship to psychopathology and premorbid adjustment. Compr Psychiatry, 39(5), 255-260.

[25]. Dickerson, F. B., Boronow, J. J., Ringel, N., \& Parente, F. (1997). Lack of insight among outpatients with schizophrenia. Psychiatr Serv, 48(2), 195-199.

[26]. Drake, R. J., \& Lewis, S. W. (2003). Insight and neurocognition in schizophrenia. Schizophr Res, 62(1-2), $165-173$.

[27]. Flashman, L. A., McAllister, T. W., Andreasen, N. C., \& Saykin, A. J. (2000). Smaller brain size associated with unawareness of illness in patients with schizophrenia. Am J Psychiatry, 157(7), 1167-1169.

[28]. Frese, F. J., 3rd. (2000). Psychology practitioners and schizophrenia: a view from both sides. J Clin Psychol, 56(11), $1413-1426$. doi: 10.1002/1097-4679(200011)56:11<1413::aid-jclp4>3.0.co;2-h

[29]. Gallagher, B. J., 3rd, Jones, B. J., McFalls, J. A., Jr., \& Pisa, A. M. (2006). Social class and type of schizophrenia. Eur Psychiatry, 21(4), 233-237. doi: 10.1016/j.eurpsy.2006.04.001

[30]. Gigante, A. D., \& Castel, S. (2004). Insight into schizophrenia: a comparative study between patients and family members. Sao Paulo Med J, 122(6), 246-251. doi: /S1516-31802004000600004

[31]. Goldberg, R. W., Green-Paden, L. D., Lehman, A. F., \& Gold, J. M. (2001). Correlates of insight in serious mental illness. J Nerv Ment Dis, 189(3), 137-145.

[32]. Goodman, C., Knoll, G., Isakov, V., \& Silver, H. (2005). Insight into illness in schizophrenia. Compr Psychiatry, 46(4), 284-290.

[33]. Granholm, E., McQuaid, J. R., McClure, F. S., Auslander, L. A., Perivoliotis, D., Pedrelli, P., . . . Jeste, D. V. (2005). A randomized, controlled trial of cognitive behavioral social skills training for middle-aged and older outpatients with chronic schizophrenia. Am J Psychiatry, 162(3), 520-529. doi: 10.1176/appi.ajp.162.3.520

[34]. Joel, D., Sathyaseelan, M., Jayakaran, R., Vijayakumar, C., Muthurathnam, S., \& Jacob, K. S. (2003). Explanatory models of psychosis among community health workers in South India. Acta Psychiatr Scand, 108(1), 66-69.

[35]. Kay, S. R., Fiszbein, A., \& Opler, L. A. (1987). The positive and negative syndrome scale (PANSS) for schizophrenia. Schizophr Bull, 13(2), 261-276.

[36]. Kemp R, D. A. (1997). Insight and compliance. In treatment compliance and therapeutic alliance in serious mental illness: The Netherlands:Harwood academic Pubilshers.

[37]. Kendler, K. S., Glazer, W. M., \& Morgenstern, H. (1983). Dimensions of delusional experience. Am J Psychiatry, $140(4), 466-469$.

[38]. Keshavan, M. S., Rabinowitz, J., DeSmedt, G., Harvey, P. D., \& Schooler, N. (2004). Correlates of insight in first episode psychosis. Schizophr Res, 70(2-3), 187-194. doi: 10.1016/j.schres.2003.11.007

[39]. Kleinman., A. (1980). Patients and healers in the context of culture: University of California.

[40]. Kurtz, M. M., Olfson, R. H., \& Rose, J. (2013). Self-efficacy and functional status in schizophrenia: relationship to insight, cognition and negative symptoms. Schizophr Res, 145(1-3), 69-74. doi: 10.1016/j.schres.2012.12.030

[41]. L.J, K., \& Korin.E. (1998). Inside knowledge:cultural construction of insight in psychosis. In X. Amador \& A. S. David (Eds.), Insight in psychosis (pp. 193-220). New York: Oxford.

[42]. Laroi, F., Fannemel, M., Ronneberg, U., Flekkoy, K., Opjordsmoen, S., Dullerud, R., \& Haakonsen, M. (2000). Unawareness of illness in chronic schizophrenia and its relationship to structural brain measures and neuropsychological tests. Psychiatry Res, $100(1), 49-58$.

[43]. Lincoln, T. M., Lullmann, E., \& Rief, W. (2007). Correlates and long-term consequences of poor insight in patients with schizophrenia. A systematic review. Schizophr Bull, 33(6), 1324-1342. doi: 10.1093/schbul/sbm002

[44]. Lloyd, K. R., Jacob, K. S., Patel, V., St Louis, L., Bhugra, D., \& Mann, A. H. (1998). The development of the Short Explanatory Model Interview (SEMI) and its use among primary-care attenders with common mental disorders. Psychol Med, 28(5), 1231-1237. 
[45]. Lysaker, P., \& Bell, M. (1994). Insight and cognitive impairment in schizophrenia. Performance on repeated administrations of the Wisconsin Card Sorting Test. J Nerv Ment Dis, 182(11), 656-660.

[46]. Lysaker, P. H., Bell, M. D., Bryson, G., \& Kaplan, E. (1998). Neurocognitive function and insight in schizophrenia: support for an association with impairments in executive function but not with impairments in global function. Acta Psychiatr Scand, 97(4), 297301 .

[47]. Lysaker, P. H., Whitney, K. A., \& Davis, L. W. (2006). Awareness of illness in schizophrenia: associations with multiple assessments of executive function. J Neuropsychiatry Clin Neurosci, 18(4), 516-520. doi: 10.1176/appi.neuropsych.18.4.516

[48]. Matsui, M., Sumiyoshi, T., Arai, H., Higuchi, Y., \& Kurachi, M. (2008). Cognitive functioning related to quality of life in schizophrenia. Prog Neuropsychopharmacol Biol Psychiatry, 32(1), 280-287. doi: 10.1016/j.pnpbp.2007.08.019

[49]. McCabe, R., \& Priebe, S. (2004). Explanatory models of illness in schizophrenia: comparison of four ethnic groups. Br J Psychiatry, 185, 25-30.

[50]. McEvoy, J. P. (2004). The relationship between insight in psychosis and compliance with medications. In D. A.S \& X. F. Amador (Eds.), Insight and psychosis (pp. 291-305). New York.

[51]. McEvoy, J. P., Applebaum, P. S., Apperson, L. J., Geller, J. L., \& Freter, S. (1989). Why must some schizophrenic patients be involuntarily committed? The role of insight. Compr Psychiatry, 30(1), 13-17.

[52]. Medalia, A., \& Thysen, J. (2010). A comparison of insight into clinical symptoms versus insight into neuro-cognitive symptoms in schizophrenia. Schizophr Res, 118(1-3), 134-139. doi: 10.1016/j.schres.2009.09.027

[53]. Mintz, A. R., Addington, J., \& Addington, D. (2004). Insight in early psychosis: a 1 -year follow-up. Schizophr Res, 67(2-3), 213217. doi: 10.1016/s0920-9964(03)00047-1

[54]. Mintz, A. R., Dobson, K. S., \& Romney, D. M. (2003). Insight in schizophrenia: a meta-analysis. Schizophr Res, 61(1), 75-88.

[55]. Monteiro, L. C., Silva, V. A., \& Louza, M. R. (2008). Insight, cognitive dysfunction and symptomatology in schizophrenia. Eur Arch Psychiatry Clin Neurosci, 258(7), 402-405. doi: 10.1007/s00406-008-0809-8

[56]. Mysore, A., Parks, R. W., Lee, K. H., Bhaker, R. S., Birkett, P., \& Woodruff, P. W. (2007). Neurocognitive basis of insight in schizophrenia. Br J Psychiatry, 190, 529-530. doi: 10.1192/bjp.bp.106.029181

[57]. Nieznanski, M., Czerwinska, M., Chojnowska, A., Walczak, S., \& Dunski, W. (2002). [Effectiveness of cognitive skills training in schizophrenia]. Psychiatr Pol, 36(5), 745-757.

[58]. Rector, N. A., \& Beck, A. T. (2002). A clinical review of cognitive therapy for schizophrenia. Curr Psychiatry Rep, 4(4), $284-292$.

[59]. Ritsner, M. S., \& Blumenkrantz, H. (2007). Predicting domain-specific insight of schizophrenia patients from symptomatology, multiple neurocognitive functions, and personality related traits. Psychiatry Res, 149(1-3), 59-69. doi: 10.1016/j.psychres.2006.01.002

[60]. Rossell, S. L., Coakes, J., Shapleske, J., Woodruff, P. W., \& David, A. S. (2003). Insight: its relationship with cognitive function, brain volume and symptoms in schizophrenia. Psychol Med, 33(1), 111-119.

[61]. Roth, R. M., Flashman, L. A., Saykin, A. J., McAllister, T. W., \& Vidaver, R. (2004). Apathy in schizophrenia: reduced fronta1 lobe volume and neuropsychological deficits. Am J Psychiatry, 161(1), 157-159.

[62]. Rusch, N., \& Corrigan, P. W. (2002). Motivational interviewing to improve insight and treatment adherence in schizophrenia. Psychiatr Rehabil J, 26(1), 23-32.

[63]. Sanz, M., Constable, G., Lopez-Ibor, I., Kemp, R., \& David, A. S. (1998). A comparative study of insight scales and their relationship to psychopathological and clinical variables. Psychol Med, 28(2), 437-446.

[64]. Saravanan, B., David, A., Bhugra, D., Prince, M., \& Jacob, K. S. (2005). Insight in people with psychosis: the influence of culture. Int Rev Psychiatry, 17(2), 83-87. doi: 10.1080/09540260500073596

[65]. Saravanan, B., Jacob, K. S., Johnson, S., Prince, M., Bhugra, D., \& David, A. S. (2007). Belief models in first episode schizophrenia in South India. Soc Psychiatry Psychiatr Epidemiol, 42(6), 446-451. doi: 10.1007/s00127-007-0186-Z

[66]. Schwartz, R. C., Cohen, B. N., \& Grubaugh, A. (1997). Does insight affect long-term impatient treatment outcome in chronic schizophrenia? Compr Psychiatry, 38(5), 283-288.

[67]. Schwartz, R. C., \& Petersen, S. (1999). The relationship between insight and suicidality among patients with schizophrenia. J Nerv Ment Dis, 187(6), 376-378.

[68]. Sevy, S., Nathanson, K., Visweswaraiah, H., \& Amador, X. (2004). The relationship between insight and symptoms in schizophrenia. Compr Psychiatry, 45(1), 16-19. doi: 10.1016/j.comppsych.2003.09.002

[69]. Shad, M. U., Muddasani, S., \& Keshavan, M. S. (2006). Prefrontal subregions and dimensions of insight in first-episode schizophrenia--a pilot study. Psychiatry Res, 146(1), 35-42. doi: 10.1016/j.pscychresns.2005.11.001

[70]. Shad, M. U., Muddasani, S., Prasad, K., Sweeney, J. A., \& Keshavan, M. S. (2004). Insight and prefrontal cortex in first-episode Schizophrenia. Neuroimage, 22(3), 1315-1320. doi: 10.1016/j.neuroimage.2004.03.016

[71]. Silver, A. L. (2003). The psychotherapy of schizophrenia: its place in the modern world. J Am Acad Psychoanal Dyn Psychiatry, 31(2), 325-341.

[72]. Simon, A. E., Berger, G. E., Giacomini, V., Ferrero, F., \& Mohr, S. (2006). Insight, symptoms and executive functions in schizophrenia. Cogn Neuropsychiatry, 11(5), 437-451. doi: 10.1080/13546800444000308

[73]. Smith, T. E., Hull, J. W., Israel, L. M., \& Willson, D. F. (2000). Insight, symptoms, and neurocognition in schizophrenia and schizoaffective disorder. Schizophr Bull, 26(1), 193-200.

[74]. Stefanopoulou, E., Lafuente, A. R., Saez Fonseca, J. A., \& Huxley, A. (2009). Insight, global functioning and psychopathology amongst in-patient clients with schizophrenia. Psychiatr Q, 80(3), 155-165. doi: 10.1007/s11126-009-9103-9

[75]. Tirupati, S., Padmavati, R., Thara, R., \& McCreadie, R. G. (2007). Insight and psychopathology in never-treated schizophrenia. Compr Psychiatry, 48(3), 264-268. doi: 10.1016/j.comppsych.2006.10.006

[76]. Tranulis, C., Corin, E., \& Kirmayer, L. J. (2008). Insight and psychosis: comparing the perspectives of patient, entourage and clinician. Int J Soc Psychiatry, 54(3), 225-241.

[77]. Valmaggia, L. R., van der Gaag, M., Tarrier, N., Pijnenborg, M., \& Slooff, C. J. (2005). Cognitive-behavioural therapy for refractory psychotic symptoms of schizophrenia resistant to atypical antipsychotic medication. Randomised controlled trial. Br $\mathrm{J}$ Psychiatry, 186, 324-330. doi: 10.1192/bjp.186.4.324

[78]. Woon, P. S., Chia, M. Y., Chan, W. Y., \& Sim, K. (2010). Neurocognitive, clinical and functional correlates of subjective quality of life in Asian outpatients with schizophrenia. Prog Neuropsychopharmacol Biol Psychiatry, 34(3), 463-468. doi: 10.1016/j.pnpbp.2010.01.014

[79]. Xavier F Amador., \& David, A. S. (2004). Insight and psychosis awareness of illness in schizophrenia and related disorders (2nd ed.).

[80]. Young, D. A., Davila, R., \& Scher, H. (1993). Unawareness of illness and neuropsychological performance in chronic schizophrenia. Schizophr Res, 10(2), 117-124. 